\begin{tabular}{|c|l|}
\hline Title & Optical response of two-level atoms with reflection geometry as a model of a quantum phase gate \\
\hline Author(s) & Oka, Hisaki; Takeuchi, Shigeki; Sasaki, Keiji \\
\hline Citation & $\begin{array}{l}\text { Physical Review A, 72, 013816 } \\
\text { https://doi.org/10.1103/PhysRevA.72.013816 }\end{array}$ \\
\hline Issue Date & 2005-07-20 \\
\hline Doc URL & http://hdl.handle.net/2115/5561 \\
\hline Rights & Copyright $\odot 2005$ A merican Physical Society \\
\hline Type & article \\
\hline File Information & PRA 72-1.pdf \\
\hline
\end{tabular}

Instructions for use 


\title{
Optical response of two-level atoms with reflection geometry as a model of a quantum phase gate
}

\author{
Hisaki Oka, ${ }^{1, *}$ Shigeki Takeuchi, ${ }^{1,2, \dagger}$ and Keiji Sasaki ${ }^{1, \$}$ \\ ${ }^{1}$ Research Institute for Electronic Science, Hokkaido University, Sapporo 060-0812, Japan \\ ${ }^{2}$ CREST, Japan Science and Technology Agency (JST), Research Institute for Electronic Science, Hokkaido University, \\ Sapporo 060-0812, Japan \\ (Received 11 March 2005; revised manuscript received 9 May 2005; published 20 July 2005)
}

\begin{abstract}
The nonlinear optical response obtained from a model system of a quantum phase gate is investigated. The model system consists of a thin infinite atomic layer of two-level atoms placed in front of a perfect reflecting mirror. The optical response obtained from the model system is semiclassically analyzed using the finite difference time domain method with the optical Bloch equations. It is shown that a nonlinear phase shift of $\pi$ is achieved when the atomic layer is placed at an antinode of the input field. This result is consistent with the theoretical result obtained from a one-dimensional atom model [H. F. Hofmann, K. Kojima, S. Takeuchi, and K. Sasaki, J. Opt. B: Quantum Semiclassical Opt. 5, 218 (2003)]. The dependence of the nonlinear phase shift on the position of the atomic layer is also studied in detail.
\end{abstract}

DOI: $10.1103 /$ PhysRevA.72.013816

PACS number(s): 42.65.-k, 32.80.-t, 03.67.Lx

\section{INTRODUCTION}

The realization of quantum devices for photonic quantum information processing is attracting a great deal of attention. Quantum phase gates (QPGs), which coherently modulate the phase of a signal photon according to a control photon, are thought to be the most essential of such devices because arbitrary unitary operation on photonic qubits can be physically realized using QPGs and simple linear optical elements [1]. Several ideas have been proposed to realize QPGs using linear optics and postevent selection [2-5], however, the low efficiency of those schemes is a critical problem.

One alternative approach is to utilize single-atom nonlinearity. Turchette et al. observed a phase shift of $\pi / 12$ with very weak c.w. light input using single atoms passing through a high-Q microcavity [6]. Based on this experiment, we have proposed a QPG device using a single atom confined in a one-sided cavity [7]. We have shown, using semiclassical analysis, that a nonlinear phase shift of $\pi$ can be obtained from our QPG device for an input light field on resonance [7], if the phase relaxation time is longer than the spontaneous decay time [8]. The change of quantum state of the photons has also been investigated using a fully quantum-mechanical treatment $[9,10]$, and the efficiency of the QPG device has been analyzed in terms of quantum state fidelity [11]. An extended investigation has recently been performed analytically by another group [12].

However, there is a limitation in the previous analyses [7-11]. These analyses are based on the theoretical model called the one-dimensional atom (1D atom), in which the cavity field is adiabatically eliminated under the bad cavity condition [7]. This means that the effect of the device structure, i.e., the position of a two-level atom in the cavity, on the nonlinear phase shift cannot be analyzed. However, to

\footnotetext{
*Email address: h-oka@es.hokudai.ac.jp

†Email address: takeuchi@es.hokudai.ac.jp

†Email address: sasaki@es.hokudai.ac.jp
}

realize a QPG device, the relation between the position of atoms and the amount of phase shift must be fully investigated.

In this paper, we investigate the above-mentioned issue using a numerical calculation of the optical Bloch equations with the finite difference time domain (FDTD) method [13]. As a first step, we investigate the optical response obtained from a model system in which a thin infinite atomic layer of two-level atoms is placed in front of a perfectly reflecting mirror. The atomic layer therefore sits in a standing wave formed in front of the mirror. We find that a nonlinear phase shift of $\pi$ can be obtained when the atomic layer is placed at an antinode of the standing wave. The dependence of the phase shift on the incident photon number is consistent with that previously reported using the $1 \mathrm{D}$ atom model [7]. We also find that the result can be well explained by destructive interference between the incident field and the field emitted from the atomic layer. We also study in detail the dependence of the nonlinear phase shift on the position of an atomic layer.

This paper is organized as follows. In Sec. II, we formulate the dynamics of the optical Bloch equations coupled with the FDTD method. In Sec. III, we analyze the effects of the position of an atomic layer on the nonlinear optical response. In Sec. IV, we summarize our results.

\section{MODEL SYSTEM AND NUMERICAL SETUP}

Figure 1 illustrates a thin infinite atomic layer, under the assumption that the atoms are stationary and uniformly distributed and do not interact with each other. When a plane wave is perpendicularly incident on the atomic layer, light emitted from the atomic layer propagates in the same plane wave mode as the incident wave owing to interference of the light emitted from each atom [14]. This system can then be reduced to a one-dimensional input-output system, and the dynamics of this system can be analyzed using onedimensional Maxwell's equations [13].

The one-dimensional Maxwell's equations with a dipole current $j_{z}$, resulting from the above reduction, are given by 


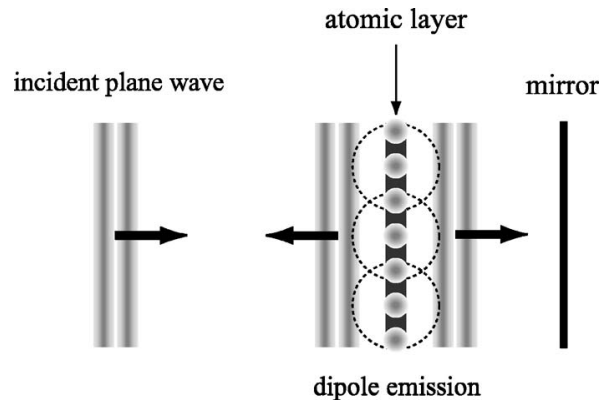

FIG. 1. Schematic of a one-dimensional Maxwell-Bloch system with reflection geometry. A plane wave is perpendicularly incident on a thin infinite atomic layer. The emitted light from the atomic layer then propagates in the same plane wave mode as the incident wave. This system can therefore be reduced to a one-dimensional input-output system with negligible output losses.

$$
\begin{gathered}
\frac{\partial E_{z}(x, t)}{\partial t}=\frac{1}{\epsilon_{0}} \frac{\partial H_{y}(x, t)}{\partial x}-\frac{1}{\epsilon_{0}} j_{z}(x, t), \\
\frac{\partial H_{y}(x, t)}{\partial t}=\frac{1}{\mu_{0}} \frac{\partial E_{z}(x, t)}{\partial x} .
\end{gathered}
$$

Note that the usual four Maxwell's equations can be reduced to the above two equations in our one-dimensional system because only the electromagnetic fields orthogonal to the $x$ axis are allowed and thus the divergences of the electric field and the magnetic field are always zero. Since the dipole current is due to the induced polarization $P_{z}$ of atoms, it can be expressed in terms of the dynamics of the polarization,

$$
j_{z}=\partial P_{z} / \partial t
$$

showing how the emitted light from the atoms in the atomic layer is coupled to Maxwell's equations through the dipole current $j_{z}$.

The dynamics of atoms in the atomic layer can be described by the optical Bloch equations of the driven twolevel atom. For a two-level atom with a ground state $|g\rangle$ and an excited state $|e\rangle$, the Bloch equations can be expressed in terms of the complex dipole operators $\hat{\sigma}_{-}=|g\rangle\langle e|$ and $\hat{\sigma}_{+}$ $=|e\rangle\langle g|$, and the inversion operator $\hat{\sigma}_{z}=(|e\rangle\langle e|-| g\rangle\langle g|) / 2$ as [15]

$$
\begin{gathered}
\frac{d}{d t}\left\langle\hat{\sigma}_{-}\right\rangle=\left(-\gamma_{\|}-i \omega_{0}\right)\left\langle\hat{\sigma}_{-}\right\rangle-i 2 \Omega\left\langle\hat{\sigma}_{z}\right\rangle, \\
\frac{d}{d t}\left\langle\hat{\sigma}_{+}\right\rangle=\left(-\gamma_{\|}+i \omega_{0}\right)\left\langle\hat{\sigma}_{+}\right\rangle+i 2 \Omega\left\langle\hat{\sigma}_{z}\right\rangle, \\
\frac{d}{d t}\left\langle\hat{\sigma}_{z}\right\rangle=-2 \gamma_{\|}\left(\left\langle\hat{\sigma}_{z}\right\rangle+\frac{1}{2}\right)-i \Omega\left(\left\langle\hat{\sigma}_{-}\right\rangle-\left\langle\hat{\sigma}_{+}\right\rangle\right),
\end{gathered}
$$

where $\gamma_{\|}$is the dipole decay rate due to spontaneous emission, $\omega_{0}$ is the resonant frequency between the two atomic levels, and $\Omega$ is the Rabi frequency given by

$$
\Omega=\frac{d_{\mathrm{eg}} E_{z}\left(x_{a}, t\right)}{\hbar},
$$

where $d_{\text {eg }}$ is the dipole moment of the atom and $x_{a}$ denotes the position of the atomic layer.

The dipole current $j_{z}$ shown in Eq. (2) can now be described using the density of polarized atoms in the atomic layer $N_{\text {atom }}$, the dipole moment of the atoms $d_{\text {eg }}$, and the expectation values of the dipole operators $\hat{\sigma}_{-}$and $\hat{\sigma}_{+}$, giving

$$
j_{z}(x, t)=N_{\text {atom }} d_{\text {eg }} \delta\left(x-x_{a}\right) \frac{d}{d t}\left(\left\langle\hat{\sigma}_{-}\right\rangle+\left\langle\hat{\sigma}_{+}\right\rangle\right) .
$$

Equation (5) describes coherent dipole emission from twolevel atoms. Equations (1) and (3) can be numerically solved by transforming them into finite difference equations. In particular, Maxwell's equations [Eq. (1)] can be solved using the FDTD method [13].

In the FDTD calculation, Maxwell's equations [Eq. (1)] are transformed into spatiotemporally finite difference equations that obey the Yee algorithm [16], $E_{z}(k \Delta x, n \Delta t)$ $\equiv E_{n}(k)$ and $H_{y}(k \Delta x, n \Delta t) \equiv H_{n}(k)$, where $k$ and $n$ denote the grid number and time step used for the FDTD calculation, respectively. The Bloch equations [Eq. (3)] are also transformed into finite difference equations in the time domain. The integration of the Maxwell-Bloch equations from the $n$th step to the $n+1$-th step is carefully performed stepwise: First we integrate Eq. (1a) except the term of $-j_{z} / \epsilon_{0}$ using the FDTD algorithm, and obtain the set of electric fields $E_{n+1}^{\prime}(k)$ for each grid number $k$. Then at the grid number $k_{a}$, where the atomic layer is set, we integrate the Bloch equations [Eq. (3)] driven only by the electric field $E_{n+1}^{\prime}\left(k_{a}\right)$, and obtain the difference values of $\left(\left\langle\hat{\sigma}_{-}\right\rangle_{n+1}-\left\langle\hat{\sigma}_{-}\right\rangle_{n}\right) / \Delta t$ and $\left(\left\langle\hat{\sigma}_{+}\right\rangle_{n+1}\right.$ $\left.-\left\langle\hat{\sigma}_{+}\right\rangle_{n}\right) / \Delta t$. Then, we calculate the dipole current $j_{z}$ using the difference equation of Eq. (5) and obtain the electric field $E_{n+1}(k)=E_{n+1}^{\prime}(k)-j_{z}(k) / \epsilon_{0}$ for each grid number $k$. Finally, we integrate Eq. (1b) with $E_{n+1}(k)$ using the FDTD algorithm, and obtain the magnetic fields $H_{n+1}(k)$ for each grid number $k$. The initial state $n=0$ is that for which all the electromagnetic fields are zero, $E_{0}=H_{0}=0$, and the two-level atom is in the ground state $\left(\left\langle\hat{\sigma}_{-}\right\rangle,\left\langle\hat{\sigma}_{+}\right\rangle,\left\langle\hat{\sigma}_{z}\right\rangle\right)=(0,0,-1 / 2)$. Throughout the numerical calculations, the expectation values of the dipole operators in the Bloch equations [Eq. (3)] are replaced with the real number parameters $\left\langle\hat{\sigma}_{x}\right\rangle=\left(\left\langle\hat{\sigma}_{-}\right\rangle\right.$ $\left.+\left\langle\hat{\sigma}_{+}\right\rangle\right) / 2$ and $\left\langle\hat{\sigma}_{y}\right\rangle=i\left(\left\langle\hat{\sigma}_{-}\right\rangle-\left\langle\hat{\sigma}_{+}\right\rangle\right) / 2$.

Figure 2 shows the input-output field calculated by the one-dimensional FDTD method in the absence of an atomic layer. A right-traveling input field in the form of a continuous wave is initially radiated from a source and is then reflected by a perfect reflecting mirror set at one end of the boundary domain [17]. The reflected light propagates to the opposite end of the boundary domain, at which we use Mur's firstorder absorbing boundary condition to eliminate the backreflection into the computational domain. On the left-hand side of the source, we can obtain an output field that consists only of the reflected component. Note that the notch appearing at the source is not due to the numerical calculation error but due to a phase lag between the output field of the traveling wave and the standing wave. This is thereforec gener- 


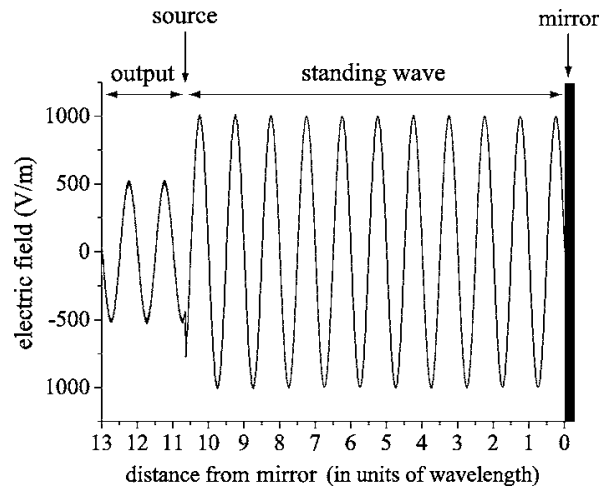

FIG. 2. Input-output field with a perfect mirror calculated by the one-dimensional FDTD method (without an atomic layer). The electric-field profile at the simulation time of $t=1.247615$ $\times 10^{-10} \mathrm{~s}$ is shown. A right-traveling input field is initially radiated from a source and then reflected by the mirror placed at the one end of the boundary domain. At the opposite end of the boundary domain we use Mur's first-order absorbing boundary condition. The output field can be obtained from the output region that consists only of the reflection component.

ally observed in the system with reflection geometry that we now consider.

For comparison with the theoretical results in Refs. [7,8], we introduce the input photon number $n_{\text {in }}$ normalized by the dipole decay rate $\gamma_{\|}$, defined as

$$
n_{\text {in }}=\frac{\bar{S} A}{\hbar \omega \gamma_{\|}}
$$

where $A$ is the atomic cross section and $\bar{S}$ is the timeaveraged Poynting vector component given by $\bar{S}=\overline{E_{z} H_{y}}$. Equation (6) shows that a mean photon number of $n_{\text {in }}$ is incident on the atomic layer during the dipole damping lifetime $\tau=1 / \gamma_{\|}$. The phase shift $\phi\left(n_{\text {in }}\right)$ for a given $n_{\text {in }}$ can then be estimated by comparing the output field for the presence of an atomic layer to the output field obtained in the absence of an atomic layer [18].

In the following FDTD calculation, a cell size of $\Delta x$ $=7.5 \times 10^{-9} \mathrm{~m}$ and a time step of $\Delta t=2.499288 \times 10^{-17} \mathrm{~s}$ are used. The frequency of the input field is set to be $\omega=2 \pi$ $\times 3.5458 \times 10^{14} \mathrm{~s}^{-1}$, which corresponds to a wavelength of $\lambda=8.45 \times 10^{-7} \mathrm{~m}$. For the atomic parameters, the resonant transition frequency is the same as the frequency of input field $\omega_{0}=\omega$ and the dipole moment is set to $d_{\mathrm{eg}}=8.0$ $\times 10^{-28} \mathrm{C} \mathrm{m}$ [19]. The spontaneous emission rate is then $2 \gamma_{\|}=8.9542 \times 10^{10} \mathrm{~s}^{-1}$, which is calculated by

$$
2 \gamma_{\|}=\frac{\left|d_{\mathrm{eg}}\right|^{2} \omega_{0}^{3}}{\pi \epsilon_{0} \hbar c^{3}}
$$

where we assume that all the atoms in the atomic layer are oriented to the same direction as the polarization of the input field. The thickness of the atomic layer is equal to one cell of $\Delta x$.

In order to compare our result to the nonlinear optical response obtained from the 1D atom model [7], the value of the density of polarized atoms $N_{\text {atom }}$ has to be carefully cho- (a)

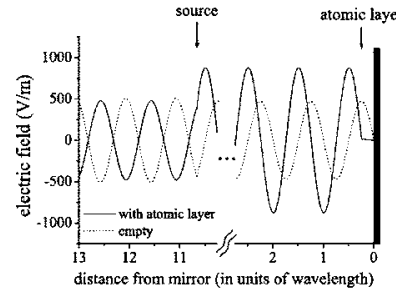

(c)

(b)

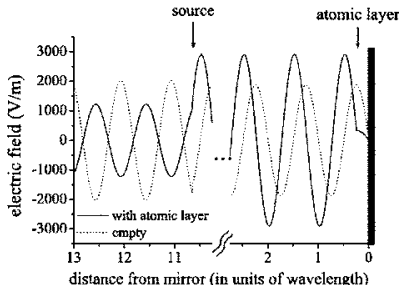

(d)

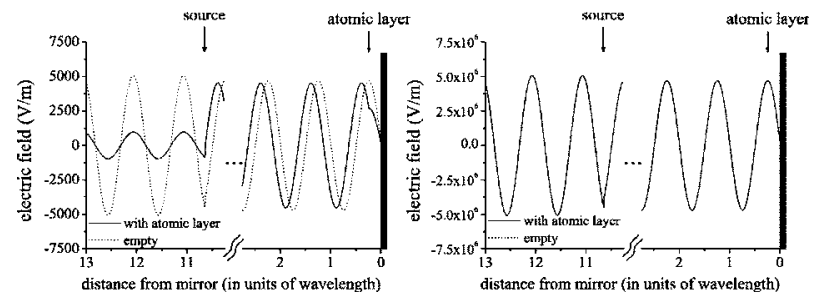

FIG. 3. Effect of input field strength on the nonlinear optical response obtained from an atomic layer for (a) $n_{\text {in }}=0.0036$, (b) $n_{\text {in }}$ $=0.057$, (c) $n_{\text {in }}=0.36$, and (d) $n_{\text {in }}=3.6 \times 10^{5}$. For all data, the electric-field profiles at the simulation time of $t=2.499204$ $\times 10^{-10} \mathrm{~s}$ are shown. The atomic layer is placed at the antinode of a standing wave closest to the mirror of the boundary domain. In order to clearly show the output and the electric field at the atomic layer, we omit the range from 2.75 to 10.25 on the horizontal axis.

sen. This can be done by using the density of polarized atoms given by the atomic cross section $A$ of a single atom,

$$
N_{\text {atom }}=\frac{1}{A \Delta x} \text { with } A=\frac{\lambda_{0}^{2}}{2 \pi},
$$

where $\lambda_{0}$ is the transition wavelength between the two atomic levels. In our case, the density of the polarized atoms is set to $N_{\text {atom }}=1.172 \times 10^{21} \mathrm{~m}^{-3}$. With Eq. (8), the dipole emission obtained from Eq. (5) can be considered that obtained from a single atom.

\section{RESULTS OF NUMERICAL CALCULATION}

Here we analyze the nonlinear optical response obtained from an atomic layer placed in a standing wave. First, in Sec. III A, we consider the case where the atomic layer is placed at an antinode of a standing wave. Then, in Sec. III B, we consider the case where the atomic layer is displaced from an antinode. Finally, in Sec. III C, we analyze in detail the dependence of the phase shift for a low input photon number on the position of the atomic layer.

\section{A. Nonlinear optical response from an atomic layer placed at an antinode}

The simplest case to consider is where an atomic layer is placed at an antinode of a standing wave. Figure 3 shows the effect of the input field strength on the nonlinear optical response obtained from an atomic layer, where the atomic layer is placed at the antinode of the standing wave closest to the mirror. As shown in Fig. 2, the output field is to the left of the source, which is set around a distance of 11 wave- 


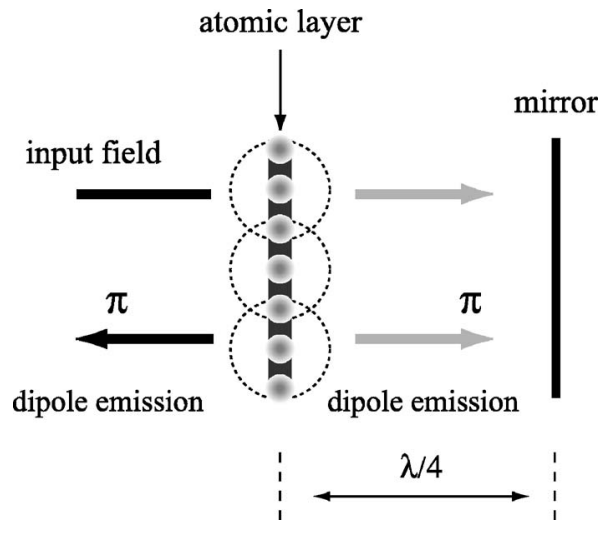

FIG. 4. Schematic of the interference between the input field and dipole emission from the atomic layer. The atomic layer is placed at a distance $\lambda / 4$ from the mirror of the end of the boundary domain. The dipole emission from the atomic layer is then in antiphase with the input field. The transmission component (gray arrows) is therefore attenuated by the destructive interference between the input field and the phase-flipped dipole emission.

lengths from the mirror. Figure 3(a) shows the optical response for an input photon number of $n_{\text {in }}=0.0036$. By comparing the output field obtained from the atomic layer (solid line) to the output field in the absence of the atomic layer (dotted line), we find that a phase shift of $\pi$ appears. Figure 3 (b) shows the optical response for $n_{\mathrm{in}}=0.057$. For an increase of the input photon number to $n_{\text {in }}=0.057$, the output field obtained from the atomic layer decreases, but the phase shift remains $\pi$. However, for a further increase of the input photon number to $n_{\text {in }}=0.36$, shown in Fig. 3(c), the phase shift becomes zero and the output field obtained from the atomic layer increases. For a larger input photon number of $n_{\text {in }}=3.6 \times 10^{5}$, as can be seen in Fig. 3(d), the output field obtained from the atomic layer is the same as the output field obtained in the absence of the atomic layer [20].

The phase shift of $\pi$ shown in Fig. 3(a) can be explained by interference between the input field and coherent dipole emission from the atomic layer (see Fig. 4). On resonance, the dipole emission is in antiphase with the local field at the atomic layer [14]. The transmission component (gray arrows in Fig. 4) is therefore attenuated by the destructive interference between the input field and the phase-flipped dipole emission. In particular, in the limit of low input photon number $\left(n_{\text {in }} \rightarrow 0\right)$, the transmission component completely vanishes and the atomic layer then acts as a perfect reflecting mirror. This can be seen in the electric field between the atomic layer and the mirror in Fig. 3(a). As a result, there is an optical path difference of $2 \lambda / 4$ between the output field obtained from the atomic layer and the output field in the absence of the atomic layer, and a phase shift of $\pi$ appears, corresponding to the optical path difference.

As the input photon number increases, however, the dipole emission from the atomic layer decreases owing to saturation of the atoms. The transmission component then does not completely vanish. The output field obtained from the atomic layer therefore consists both of the reflection component of dipole emission and the transmission component re- (a)

(b)
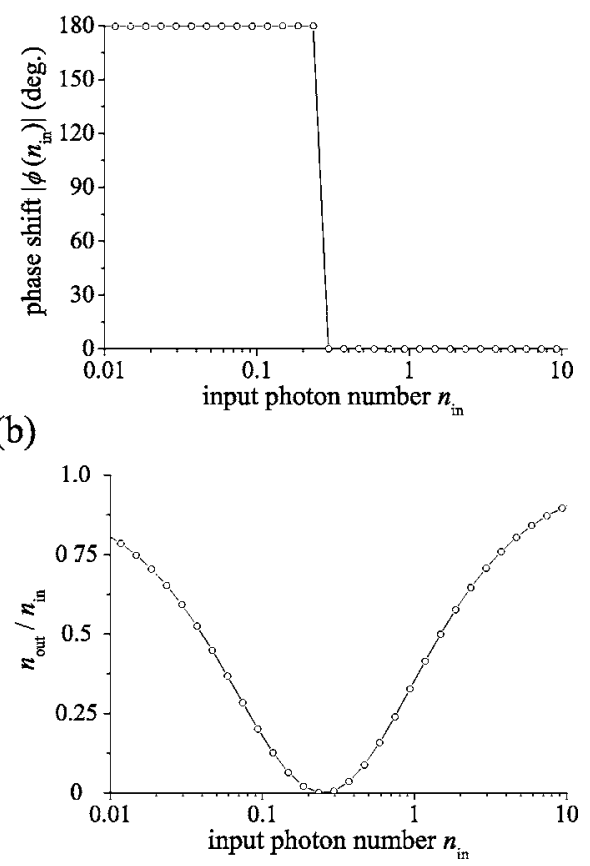

FIG. 5. (a) Nonlinear phase shift and (b) coherent output photon ratio $n_{\text {out }} / n_{\text {in }}$ obtained for an atomic layer placed at an antinode. For all data, the calculation step is $10^{7}$. The solid lines are guides for the reader's eye.

flected by the mirror. When the reflected transmission component dominates the reflection component of dipole emission, a nonlinear phase change from a phase shift of $\pi$ to a phase shift of zero occurs, as can be seen in Figs. 3(b) and $3(\mathrm{c})$. In the limit of large input photon number $\left(n_{\text {in }}\right.$ $\rightarrow \infty$ ), atoms are completely saturated and the effect of dipole emission is negligibly small, as seen in Fig. 3(d).

In order to identify this dependence of the nonlinear phase shift and the output field strength on $n_{\text {in }}$, it is useful to separate the output field into a phase shift $\phi\left(n_{\text {in }}\right)$ and a coherent output photon number $n_{\text {out }}$. The output photon number $n_{\text {out }}$ is obtained from Eq. (6) by using the electromagnetic fields at the output field. Figure 5(a) shows the dependence of the nonlinear phase shift $\phi\left(n_{\text {in }}\right)$ on $n_{\text {in }}$. For $n_{\text {in }}$ lower than $1 / 4$, the phase shift is $\pi$. When $n_{\text {in }}$ is $1 / 4$, the phase shift suddenly becomes zero and remains zero for $n_{\text {in }}$ larger than $1 / 4$. This phase switching at $n_{\mathrm{in}}=1 / 4$ is consistent with the theoretical result obtained from the 1D atom model in Ref. [7].

Figure 5(b) shows the dependence of the output photon ratio $n_{\text {out }} / n_{\text {in }}$ on $n_{\text {in }}$. In the limit of low input photon number $\left(n_{\text {in }} \rightarrow 0\right)$, the output field consists only of the reflection component of dipole emission, and $n_{\text {out }} / n_{\text {in }}$ approaches one, though this is not shown in Fig. 5(b). As $n_{\text {in }}$ increases, the reflected transmission component increases and $n_{\text {out }} / n_{\text {in }}$ decreases. For $n_{\text {in }}$ lower than $1 / 4$, the phase-flipped dipole emission still dominates the reflected transmission component. When $n_{\text {in }}$ is $1 / 4$, the amplitudes of the reflected transmission component and the phase-flipped dipole emission are exactly equal, and the coherent output is totally eliminated by the destructive interference between these two components. As a result, $n_{\text {out }} / n_{\text {in }}$ becomes zero [21]. For a further 
(a)

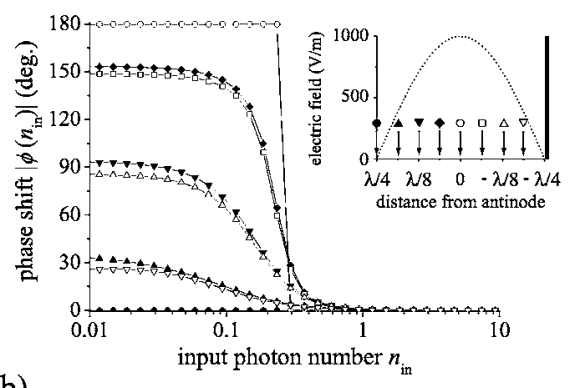

(b)

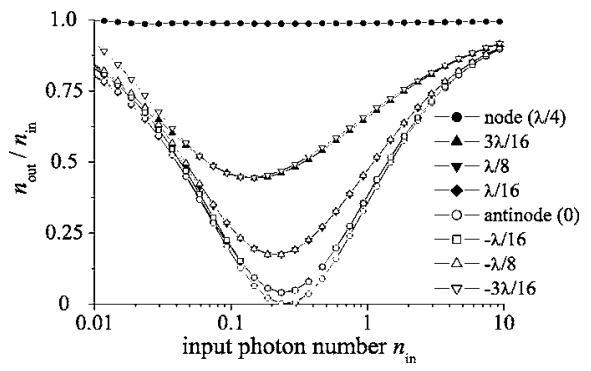

FIG. 6. (a) Nonlinear phase shift and (b) coherent output photon ratio $n_{\text {out }} / n_{\text {in }}$ obtained for an atomic layer displaced from an antinode. The positions of the atomic layer are described by the distance from the antinode of the standing wave closest to the mirror (the minus sign denotes that the atomic layer is displaced towards to the mirror). For all data, the calculation step is $10^{7}$. The solid lines are guides for the reader's eye.

increase of input photon number $\left(n_{\text {in }}>1 / 4\right)$, however, the reflected transmission component dominates the dipole emission, and $n_{\text {out }} / n_{\text {in }}$ increases. In the limit of large input photon number $\left(n_{\text {in }} \rightarrow \infty\right), n_{\text {out }} / n_{\text {in }}$ approaches one, where the output field consists only of the reflected transmission component.

\section{B. Effects of atomic layer position on the nonlinear optical response}

We now consider the case when an atomic layer is displaced from an antinode of a standing wave. Figure 6(a) shows the dependence of the nonlinear phase shift $\phi\left(n_{\text {in }}\right)$ on input photon number $n_{\text {in }}$. As shown in the inset in Fig. 6(a), the atomic layer is displaced from an antinode at intervals of $\lambda / 16$. For comparison, the nonlinear phase shift with the atomic layer at the antinode, which is shown in Fig. 5(a), is also plotted as circles $(\bigcirc)$. For each position of the atomic layer, $\phi\left(n_{\text {in }}\right)$ is larger for the lower input photon number. Specifically, for $n_{\text {in }}=0.01$, the phase shifts for positions near the antinode ( and $\square$ ) decrease to $|\phi(0.01)| \approx 150^{\circ}$. The further the position of the atomic layer is displaced from the antinode, the smaller is the absolute value of the phase shift $|\phi(0.01)|$. As $n_{\text {in }}$ increases, $\phi\left(n_{\text {in }}\right)$ gently decreases to zero, in contrast to the phase shift for the antinode. For the atomic layer placed at the node $(\bullet), \phi\left(n_{\text {in }}\right)$ is always zero. Thus $\phi\left(n_{\text {in }}\right)$ strongly depends on the position of the atomic layer. We will consider in detail the mechanism of this dependence in Sec. III C.

Figure 6(b) shows the dependence of the output photon ratio $n_{\text {out }} / n_{\text {in }}$ on $n_{\text {in }}$. For each position of the atomic layer, except at the node, $n_{\text {out }} / n_{\text {in }}$ increases to one for both small and large $n_{\text {in. }}$. However, it is interesting to note that $n_{\text {out }} / n_{\text {in }}$ for low input photon number is almost the same for each position of the atomic layer. A remarkable effect of atomic layer position on $n_{\text {out }} / n_{\text {in }}$ can be seen around $n_{\text {in }}=1 / 4$. Specifically, $n_{\text {out }} / n_{\text {in }}$ increases as the distance between the atomic layer and the antinode increases. This recovery of coherent output results from a decrease of the destructive interference effect in the output field, as discussed in Sec. III A.

As the examples in Figs. 6(a) and 6(b) show, a large phase shift and high output efficiency can be obtained for low input photon number $\left(n_{\text {in }} \ll 1\right)$. In particular, for applications to nonlinear optical devices such as QPGs, the phase shift obtained for low input photon number is important because the maximal conditional phase shift obtained by varying $n_{\text {in }}$ is equal to the phase shift for low input photon number. In the following section, we discuss in detail the dependence of the phase shift for low input photon number on the atomic layer position.

\section{Dependence of the phase shift for low input photon number on the atomic layer position}

As shown in Fig. 6, a phase shift of $\pi$ is obtained for the atomic layer placed at the antinode, and it decreases as the displacement of the atomic layer from the antinode increases, becoming zero at the node. This result cannot be easily explained by the local field strength of a standing wave in the absence of an atomic layer. In the case when the atomic layer is placed at an antinode, as discussed in Sec. III A, the dipole emission from the atomic layer completely destructively interferes with the input field, and the transmission component is then negligibly small. However, as the atomic layer is displaced from the antinode, the transmission component cannot completely vanish, and we therefore take into account the reabsorption-reemission process of atoms induced by the transmission component reflected by a perfect mirror. Here we clarify the effect of this reabsorption-reemission process on the phase shift by comparing the result in Fig. 6(a) to the phase shift obtained from the dipole emission dependent only on the local field strength of the standing wave, that is, the phase shift without the reabsorption-reemission process.

Figure 7 shows the phase shift $\left|\phi\left(n_{\text {in }}\right)\right|$ for a low input photon number of $n_{\text {in }}=0.0036$ as a function of atomic layer position. The atomic layer is displaced from an antinode at intervals of $\lambda / 32$. The dotted line is $|\phi|$ obtained theoretically from the output field without reabsorption-reemission of atoms. $|\phi|$ is obtained by using the complex amplitude $E_{\text {in }}$ of the input field and the local electric-field strength $\alpha$ $=\cos (2 \pi x / \lambda)$ of the standing wave ( $x=0$ at the antinode $)$, as

$$
\begin{gathered}
|\phi|=\left|\arg \left[\frac{E_{\text {out }}}{E_{\text {empty }}}\right]\right| \\
\text { with } E_{\text {out }}=-\alpha E_{\text {in }} e^{-i \delta}-\left(E_{\text {in }}-\alpha E_{\text {in }}\right), \\
E_{\text {empty }}=-E_{\text {in }},
\end{gathered}
$$

where the first term of the right-hand side in Eq. (9a) is the reflection component of dipole emission and the second term 


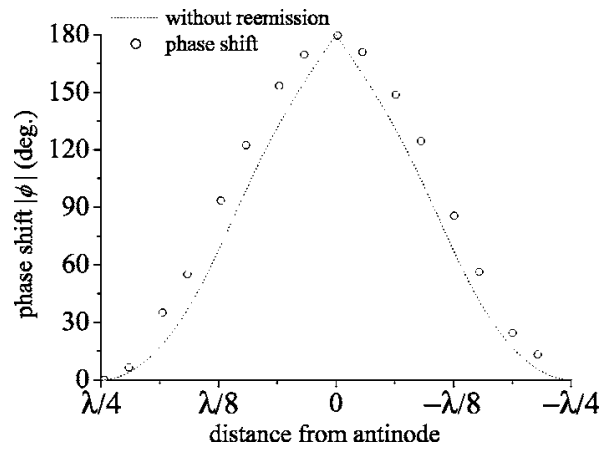

FIG. 7. Dependence of phase shift for low input photon number on atomic layer position. The phase shift $|\phi|$ for an input photon number of $n_{\text {in }}=0.0036$ is used. For all data, the calculation step is $10^{7}$. The dotted line is the phase shift theoretically obtained from the output field without the reemission component.

is the transmission component reflected by the mirror (see Fig. 4). $E_{\text {empty }}$ is the output field obtained in the absence of the atomic layer, and $\delta$ denotes the phase lag of the optical path difference between the atomic layer and the mirror, given by $\delta=2\left(\pi / 2+2 \pi x_{a} / \lambda\right)$. As can be seen in Fig. 7, at the antinode and the node where reabsorption-reemission is negligible, the phase shifts $|\phi(0.0036)|$ and $|\phi|$ are equal. However, at points other than at the antinode and the node, $|\phi(0.0036)|$ is always larger than $|\phi|$, though the shape of the function $|\phi|$ is similar to $|\phi(0.0036)|$. The phase difference between $|\phi(0.0036)|$ and $|\phi|$ is largest at the middle point of the antinode and the node, where the phase difference is $\sim 15^{\circ}$. Thus the phase shift for low input photon number is strongly modified by the reabsorption-reemission process of atoms, which cannot be explained only by the local field strength of the standing wave in the absence of the atomic layer.

In general, theoretical analysis of the phase shift with reabsorption-reemission is complicated because the reemission component is also reflected by the mirror and induces subsequent reemission. This kind of autoregressive problem may be approximately solved by using a self-consistent field, i.e., the mean field approximation. However, the FDTD method with the optical Bloch equations can automatically calculate the reabsorption-reemission process, even for a sys- tem with a complex device structure. FDTD analysis is therefore useful for analyzing the effects of atomic layer position on the optical response obtained from devices based on $1 \mathrm{D}$ atom nonlinearity.

\section{CONCLUSION}

Using the finite difference time domain method with the optical Bloch equations, we have investigated the nonlinear optical response obtained from a thin infinite atomic layer placed in front of a perfect reflecting mirror. We found that a nonlinear phase shift of $\pi$ appears when the atomic layer is placed at an antinode of a standing wave. This is consistent with the nonlinear optical response calculated using a 1D atom model [7]. We also analyzed the effect of the position of the atomic layer on the nonlinear phase shift. As the distance between the positions of the atomic layer and an antinode increases, the nonlinear phase shift decreases. In particular, the phase shift obtained for low input photon number $\left(n_{\text {in }} \ll 1\right)$ strongly depends on the interference modified by the reabsorption-reemission process of atoms rather than the local field strength at the atomic layer. We also found that the output photon ratio $n_{\text {out }} / n_{\text {in }}$ for low input photon number $n_{\text {in }}$ is almost independent of the displacement of the atomic layer from an antinode.

In this analysis, we investigated the nonlinear optical response to a coherent c.w. input field in order to compare with the results in previous works $[7,8]$. However, it is easy to expand the analysis to a system driven by a pulsed input field. It may then be interesting to consider the implications of our results for the nonlinear optical response to one- and two-photon wave functions discussed in Refs. [9,10]. FDTD calculations of higher dimensions and for a cavity structure may help to evaluate the performance of optical devices.

\section{ACKNOWLEDGMENTS}

We would like to thank H. F. Hofmann and H. Fujiwara for their helpful advice. Part of this work was supported by the program "R\&D support scheme for funding selected IT proposals" of the Ministry of Public Management, Home Affairs, Posts and Telecommunications.
[1] I. L. Chuang and Y. Yamamoto, Phys. Rev. A 52, 3489 (1995).

[2] E. Knill, R. LaOamme, and G. J. Milburn, Nature (London) 409, 46 (2001).

[3] M. Koashi, T. Yamamoto, and N. Imoto, Phys. Rev. A 63, 030301(R) (2001).

[4] J. L. O'Brien, G. J. Pryde, A. G. White, T. C. Ralph, and D. Branning, Nature (London) 426, 264 (2003).

[5] H. F. Hofmann and S. Takeuchi, Phys. Rev. A 66, 024308 (2002).

[6] Q. A. Turchette, C. J. Hood, W. Lange, H. Mabuchi, and H. J. Kimble, Phys. Rev. Lett. 75, 4710 (1995).

[7] H. F. Hofmann, K. Kojima, S. Takeuchi, and K. Sasaki, J. Opt.
B: Quantum Semiclassical Opt. 5, 218 (2003).

[8] H. Oka, H. F. Hofmann, S. Takeuchi, and K. Sasaki, Jpn. J. Appl. Phys., Part 1 43, 7495 (2004).

[9] K. Kojima, H. F. Hofmann, S. Takeuchi, and K. Sasaki, Phys. Rev. A 68, 013803 (2003).

[10] H. F. Hofmann, K. Kojima, S. Takeuchi, and K. Sasaki, Phys. Rev. A 68, 043813 (2003).

[11] K. Kojima, H. F. Hofmann, S. Takeuchi, and K. Sasaki, Phys. Rev. A 70, 013810 (2004).

[12] K. Koshino and H. Ishihara, Phys. Rev. Lett. 93, 173601 (2004).

[13] R. W. Ziolkowski, J. M. Arnold, and D. M. Gogny, Phys. Rev. 
A 52, 3082 (1995).

[14] A. V. Durrant, Am. J. Phys. 44, 630 (1976).

[15] D. F. Walls and G. J. Milburn, Quantum Optics (SpringerVerlag, Berlin, 1995). In this paper, the optical Bloch equations described in the Cartesian coordinates are used in order to simplify the numerical calculation.

[16] K. S. Yee, IEEE Trans. Antennas Propag. AP-14, 302 (1966).

[17] This right-traveling input field is obtained by simultaneously yielding the electric field $E$ and the magnetic field $H$ in the FDTD calculation.

[18] Homedyne detection is used in order to estimate the phase shift: J. W. Noh, A. Fougeres, and L. Mandel, Phys. Rev. A
45, 424 (1992).

[19] This value of dipole moment is about ten times as large as the common values of quantum dots. This is for the purpose of shortening the computational time and has no effect on the calculation results.

[20] Discrepancy of electric-field profiles between Figs. 2 and 3(d) results simply from the difference of simulation time between them.

[21] It should be noted that atom saturation also induces an increase of incoherent dipole emission [7]. However, this incoherent emission does not appear in our numerical calculation based on the semiclassical treatment. 